\title{
The Dynamics of Fidelity over the Time Course of Long-Term Memory
}

\author{
Kimele Persaud (kimele.persaud@ rutgers.edu) \\ Pernille Hemmer (pernille.hemmer@rutgers.edu ) \\ Rutgers University, Department of Psychology \\ 152 Frelinghuysen Rd, Piscataway, NJ, 08854 USA
}

\begin{abstract}
Author Note
This data was previously presented at the annual meeting of the Society for Mathematical Psychology and at the annual meeting of the Cognitive Science Society.
\end{abstract}

\section{Corresponding Author}

Kimele Persaud

Office phone: 848-445-8921

Fax: 732-445-2263

email: kimele.persaud@rutgers.edu 


\begin{abstract}
Bayesian models of cognition assume that prior knowledge about the world influences judgments. Recent approaches have suggested that the loss of fidelity from working to long-term (LT) memory is simply due to an increased rate of guessing (e.g. Brady et al. 2013). That is, recall is the result of either remembering (with some noise) or guessing. This stands in contrast to Bayesian models of cognition while assume that prior knowledge about the world influences judgments, and that recall is a combination of expectations learned from the environment and noisy memory representations. Here, we evaluate the time course of fidelity in LT episodic memory, and the relative contribution of prior category knowledge and guessing, using a continuous recall paradigm. At an aggregate level, performance reflects a high rate of guessing. However, when aggregate data is partitioned by lag (i.e., the number of presentations from study to test), or is un-aggregated, performance appears to be more complex than just remembering with some noise and guessing. We implemented three models: the standard remember-guess model, a three-component remember-guess model, and a Bayesian mixture model and evaluated these models against the data. The results emphasize the importance of taking into account the influence of prior category knowledge on memory.
\end{abstract} Keywords: Episodic memory; prior knowledge and expectations; Remember-Guess models; Bayesian mixture models. 


\section{Introduction}

An important question for memory is whether category knowledge biases performance, and whether an influence of category knowledge changes as a function of the fidelity of memory.

Recent work in visual working memory has suggested that when recalling stimulus features, observers either remember the episodic information with some noise or guess (Brady, Konkle, Gill, Oliva, \& Alvarez, 2013; Zhang and Luck, 2008). Zhang and Luck found that fidelity is fixed once capacity of visual working memory is reached, but that the guessing rate changes. The resulting error distributions are well fit by a mixture of a Gaussian-like (remembering with some noise) and uniform distribution (guessing). They argued that observers remember continuous feature values and are not biased by categorization of those values. Importantly, a finding of category bias would suggest an intermediating step between remembering and random guessing. Such a bias was found by Bae and colleagues, establishing that category biases originate in perception and are reflected in visual working memory (Bae, Olkonnen, Allred, \& Flombaum, 2015).

Several extensions to the original remember-guess model have been implemented to account for additional factors that influence visual short-term and working memory performance (e.g., Bays, Catalao, \& Husain, 2009; Bays, Wu, \& Husain, 2011; van den Berg, Shin, Chou, George, \& Ma, 2012). For example, the variable-precision model (VP; van den Berg, et al, 2012) postulates variability in the precision with which items are encoded in working memory. The resulting error distribution is a mixture of many von Mises distributions (as opposed to the one memory component in the remember-guess model), to account for residual noise in memory that the standard model cannot fit. Other proposed models incorporate task-based components, such 
as "misassociation" or "misbinding" parameters to extend the standard remember-guess model (Bays, Catalao, \& Husain, 2009; Bays, Wu, \& Husain, 2011).

Although these models provide substantial revisions to the original, it is important to note that they are grounded in visual short-term and working memory. Relatively few studies have sought to apply the remember-guess framework to understanding long-term episodic memory. One such application by Brady and colleagues (2013) showed that there is a loss of fidelity from working into long-term (LT) memory. They argued that this decrease in fidelity is due to an increased rate of guessing, without addressing other factors that impact long-term memory.

The remember-guess model stands in direct contrast to a number of Bayesian cognitive models which assume that LT memory is an integration of expectations learned from the environment with noisy memory representations (e.g., Hemmer \& Steyvers, 2009; Hemmer, Tauber \& Steyvers, 2015; Hemmer, Persaud, Kidd, \& Piantadosi, 2015). These models are pervasive in cognition in general, and in specific domains including categorization (e.g., Huttenlocher, Hedges \& Vevea, 2000), generalization (e.g. Griffiths \& Tenenbaum, 2006), semantic memory (Hemmer \& Steyvers, 2009b; Steyvers, Griffiths, \& Dennis, 2006), and episodic memory (Shiffrin \& Steyvers, 1997; Steyvers \& Griffiths, 2008).

Bayesian models of cognition propose a tradeoff between the fidelity of memory content and the influence of prior expectations. When the fidelity of the episodic trace is high, for example, as in visual short-term memory, there is minimal noise and potentially little influence of prior expectations. As fidelity decreases in working and LT memory, whether as a function of time or errors in retrieval, the influence of prior expectations would increase.

At an aggregate level, however, the error distributions resemble a combination of precise and imprecise memory, which might appear only to be remembered content and guessing, effectively 
masking underlying stages between the two. Prior expectation is a potential factor that might compensate for decreasing memory fidelity at the stage between precise memory and random guessing. In point of fact, Donkin and colleagues (2014) showed model-based evidence from visual short-term memory positing three discrete states of memory: One, a state based on perceptual memory and high precision, two, due to memory decay from perception, a state with intermediate precision based on verbal labeling, and three, guessing. Here, we seek to compare the performance of models that have been employed to characterize long-term memory, namely the remember-guess model (Brady et al, 2013) and Bayesian models of long-term memory (e.g., Hemmer \& Steyvers, 2009; Persaud \& Hemmer, 2014).

In the present work, we explore what happens to the precision of memory over time. Partitioning performance by the number of intervening trials between study and test (i.e., lag) allows for the systematic assessment of the time course of fidelity in LT episodic memory. To the best of our knowledge, this paper gives the first analysis of free recall by lag in an effort to understand the relative contributions of prior knowledge and guessing. We also investigate if category bias, indicative of the employment of prior knowledge, is a mechanism by which LT memory can be filled in, before individuals resort to random guessing. If this is the case, then performance at intermediate lags, consistent with the Bayesian assumption, should reflect the influence of category knowledge on noisy episodic representations. Such an influence is generally observed as a regression to the mean effect. We implement three models: the standard remember-guess $(\mathrm{RG})$ model, a three component remember-guess (3CRG) model, which assumes two levels of precision in memory and a Bayesian mixture (BM) model. We also conduct model comparisons as a function of lag.

\section{Memory for color: Overview of Experiment}


Our objective was to determine the contribution of prior expectations to LT episodic memory and assess the resulting time course of errors. We developed a novel experimental approach for assessing free recall for color, where participants generated recalled hue values using a continuous color wheel, and with interleaved trials of random lag lengths between study and test.

\subsection{Method}

\subsubsection{Participants}

Sixty-one Introductory Psychology undergraduate students at Rutgers University participated for course credit or $\$ 10$ compensation. In condition 1 (Label first Condition) $\mathrm{N}=18$. In condition 2 (Label after Condition) $\mathrm{N}=5$. In condition 3 (No Label Condition) $\mathrm{N}=38$. All participants reported having normal color vision. No individual participated in more than one condition.

\subsubsection{Materials}

The stimuli consisted of 48 arbitrary shapes uniformly filled with 48 colors sampled from the winHSL240 (hue, saturation, and luminance) color space. See Figure 1 for sample stimuli. The shapes were selected such that there was little prior association of any color to the shapes, that is, the study set did not result in canonical pairings such as yellow stars or red hearts. The purpose of the shapes was to cue subjects on test trials to recall the fill-color of the shape. Colors were sampled in 48 equally sized steps along the full hue range, based on the ability to perceptually differentiate two sequential colors in the range. Saturation and luminance were held constant at $100 \%$ and $50 \%$, respectively. The shapes and colors were paired randomly, and pairings were randomized across participants. Each shape and color was studied only once.

\subsubsection{Procedure}


Participants were shown a continuous study-test sequence of color filled shapes. Shapes were presented one at a time at the center of the computer screen for 2 seconds. Participants were told to study the color of each shape, as they would be asked to recall the color of the shapes. Test trials were randomly interleaved between study trials, resulting in lags of varying length. This sequence of lag was obtained by first randomly permuting the order of study trials, and then interleaving test trials, with the condition that for a test trial to occur, the corresponding study item must have occurred first. Figure 1 provides an example of the experimental procedure for a lag of 1 and a lag of 3 trials, as well as an illustration of the interleaved study test sequence.

On a test trial, a shape from a previous study trial, but filled with gray, was presented as a cue and participants were prompted to make several responses. In all three test conditions, participants first completed a recognition task for the shape. In the two label conditions, participants were asked to provide a verbal label for the color of the shape either before or after recreating the shape color (this question was posed regardless of their response to the recognition
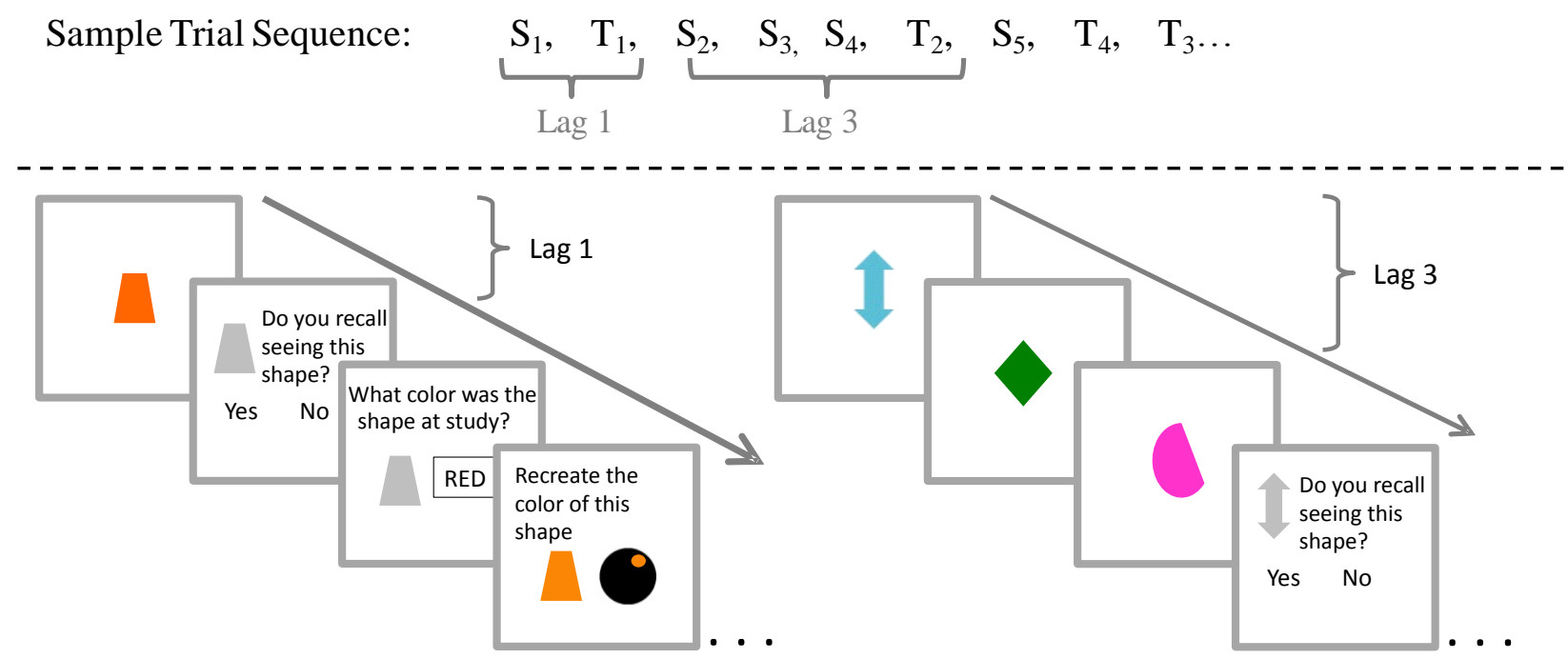

Figure 1. Sample study/test sequence by lag. Lag 1, participants study a shape, followed by a series of memory questions related to the color of the shape. Lag 3, participants study a sequence of three colored shapes, before being asked a series of memory questions related to the color of the cued shape - here, the first of the three shapes studied. 
question). Participants typed responses into a text box and pressed "enter" to continue. In condition 3, participants did not provide a verbal label. In all three conditions, participants were then asked to recreate the studied color of the shape using a continuous color wheel. The color wheel was covered by a black mask, and was randomly rotated by 90 degrees on every test trial. Participants clicked on the wheel to fill the shape with the underlying color. Test trials were selfpaced.

\subsection{Results}

For analysis, and to accommodate the use of von Mises distributions in the models, hue values were converted from the winHSL240 color space to degrees.

The primary purpose of the three labeling conditions was to check that the explicit label generation did not alter the influence of category knowledge. We find no real differences between the label versus no-label conditions, and for the purposes of analysis, data is pooled across all three conditions (see Appendix Table A1 for parameter estimation for the label versus no-label conditions).

2.2.1 Lag Analysis To measure the time course of fidelity in LT memory, the data was partitioned by lag and each resulting error distribution was analyzed. Since lag intervals encompassed participant responses which were self-paced, lag intervals varied both across trials (with the same lag) and across participants. For an approximation of the correspondence of lag intervals to units of time, we calculated the average study plus response time for each condition and collapsed across conditions. The results were as follows: Label First: $M=16.2 \mathrm{~s}, \mathrm{SD}=9.0 \mathrm{~s}$, $\mathrm{MO}=10.0 \mathrm{~s}$; Label Last: $\mathrm{M}=18.5 \mathrm{~s}, \mathrm{SD}=8.2 \mathrm{~s}, \mathrm{MO}=10.0 \mathrm{~s}$; No label: $\mathrm{M}=11.2 \mathrm{~s}, \mathrm{SD}=7.1 \mathrm{~s}, \mathrm{MO}=$ 5.0s; All conditions: $\mathrm{M}=13.3 \mathrm{~s}, \mathrm{SD}=8.3 \mathrm{~s}, \mathrm{MO}=9.0 \mathrm{~s}$. A Pearson's correlation revealed a strong positive correlation between lag and response times $(\mathrm{r}=0.7, \mathrm{p}<.000)$. 


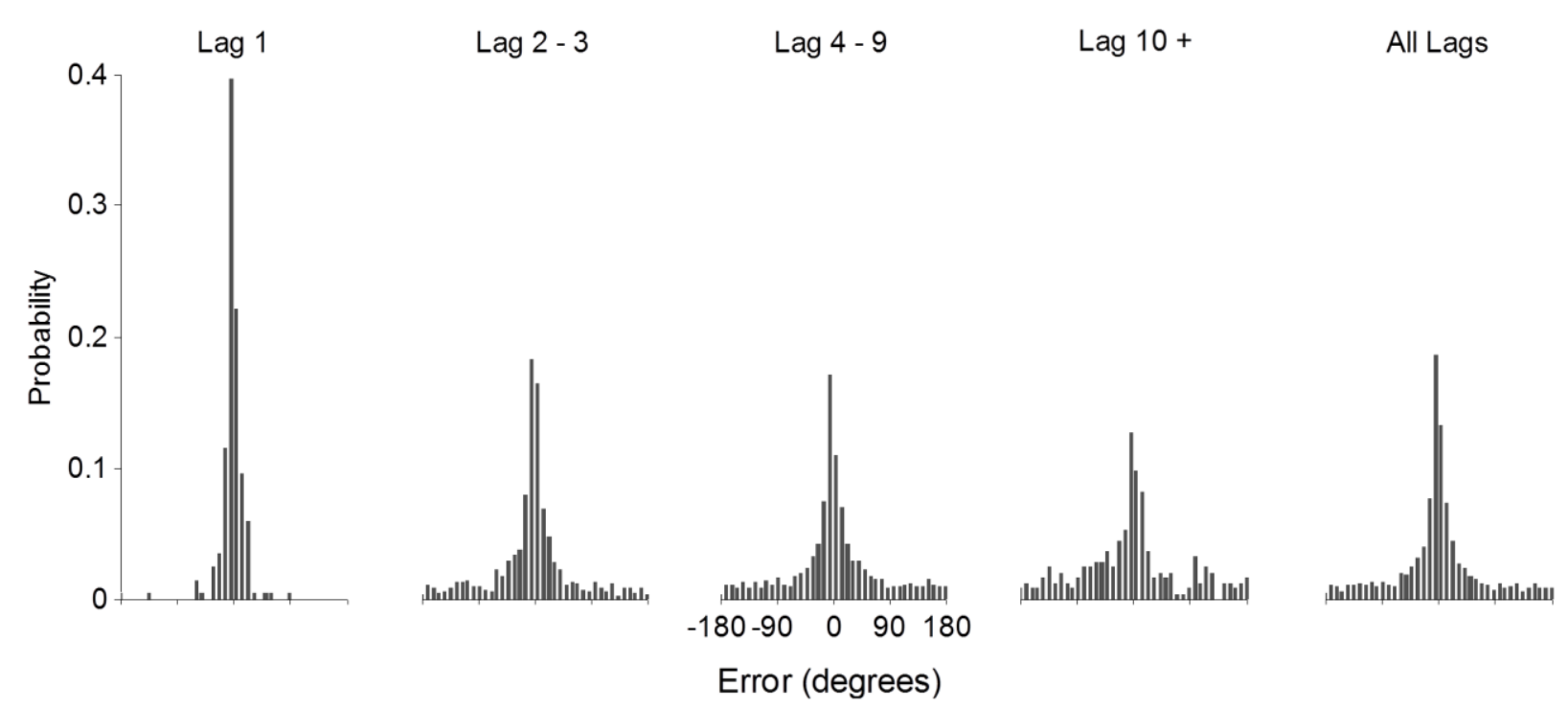

Figure 2. Histograms of errors as a function of lag: lag 1, lag 2-3, lag 4-9, lag 10+, as well as the error distribution for all lags.

Initially, all lag groups were examined separately, but then grouped based on a meaningful progression in the parameter contributions. This was done both for visual clarity, and in order to increase the "speed" of model fitting. See Appendix Table A2 for fits to all lags. Figure 2, from left to right shows the error distributions for lag 1, 2-3, 4-9, 10+, and the aggregate of all lags. The error distributions reveal that the fidelity of memory is quite high at lag 1 . This is evidenced by the tight grouping of responses around 0 error and virtually no responses past 50 degrees of error. For the remaining lags, memory fidelity is not as high as in lag 1, but does appear to be stable over time. However, there is also an increased frequency of responses past 50 degrees of error (i.e., increased rate of guessing).

Partitioning the data by lag shows a progression in the decrease of fidelity, and corresponding increase in the rate of guessing, that cannot be discerned from an aggregate error distribution. Furthermore, in the aggregate error distribution (Figure 2, 'All lags' panel), the center portion of the error distribution — which under the remember-guess model is characterized 
by a single Gaussian distribution — appears to have both a sharp peak as well as broad 'shoulders' suggesting multiple components. However, a visual inspection of the error distributions by lag is insufficient to determine whether the composition of the error distribution is strictly that of remembering and guessing, or if there are additional factors at play.

2.2.2 Recall bias To assess bias in recall we calculated the difference between the hue value recalled and the hue value studied. Figure 3, top left panel, shows study hue values as a function of bias. The square boxes illustrate the bias for each studied value scaled by the frequency at which the response was given across participants. Each square box is colored with the true recalled hue value given for each studied value. All responses to a particular studied value form a straight horizontal line, and correctly recalled hue values lie vertically at the zero-bias line of the $\mathrm{x}$-axis. The results shows regression to the mean effects for several color categories, where accuracy is greatest closer to the mean of the categories and hue values greater than the category mean are predominately underestimated (to the left of the zero bias line in Figure 3, top middle panel), while hue values less than the mean are overestimated (to the right of the zero baseline in Figure 3, top middle panel). Notably, there is an asymmetry in the distribution of responses around the zero bias line within color categories. When there is a large mass of values to the left of the zero bias line (underestimation), there are very few values to the immediate right, and vice versa. This results in strong diagonal bands (tilted on the vertical axis) within categories that are not merely a result of how the data are plotted. We take the asymmetry to indicate regression to distinct categories, and evidence of an influence of prior category knowledge on memory (Hemmer, Tauber, \& Steyvers, 2015; Hemmer \& Steyvers 2009a; Huttenlocher, Hedges, \& 


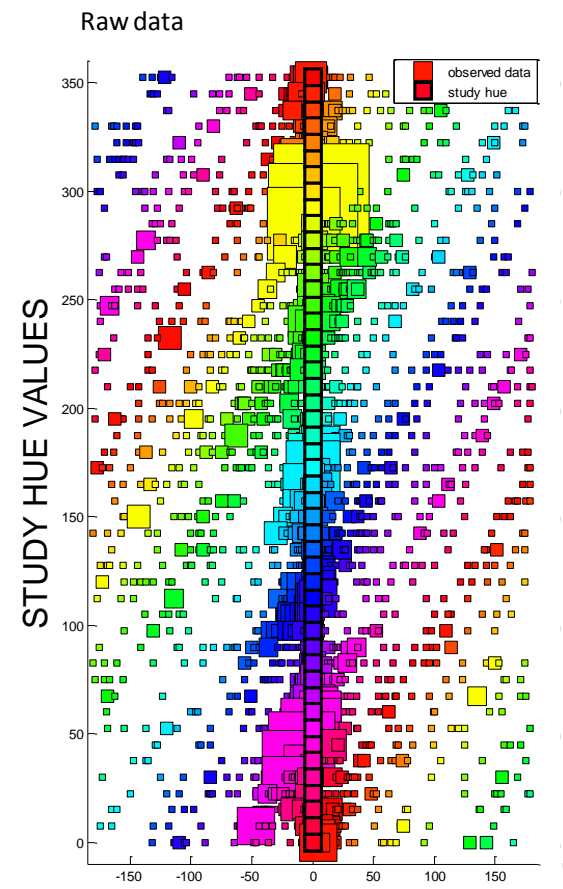

Asymmetry
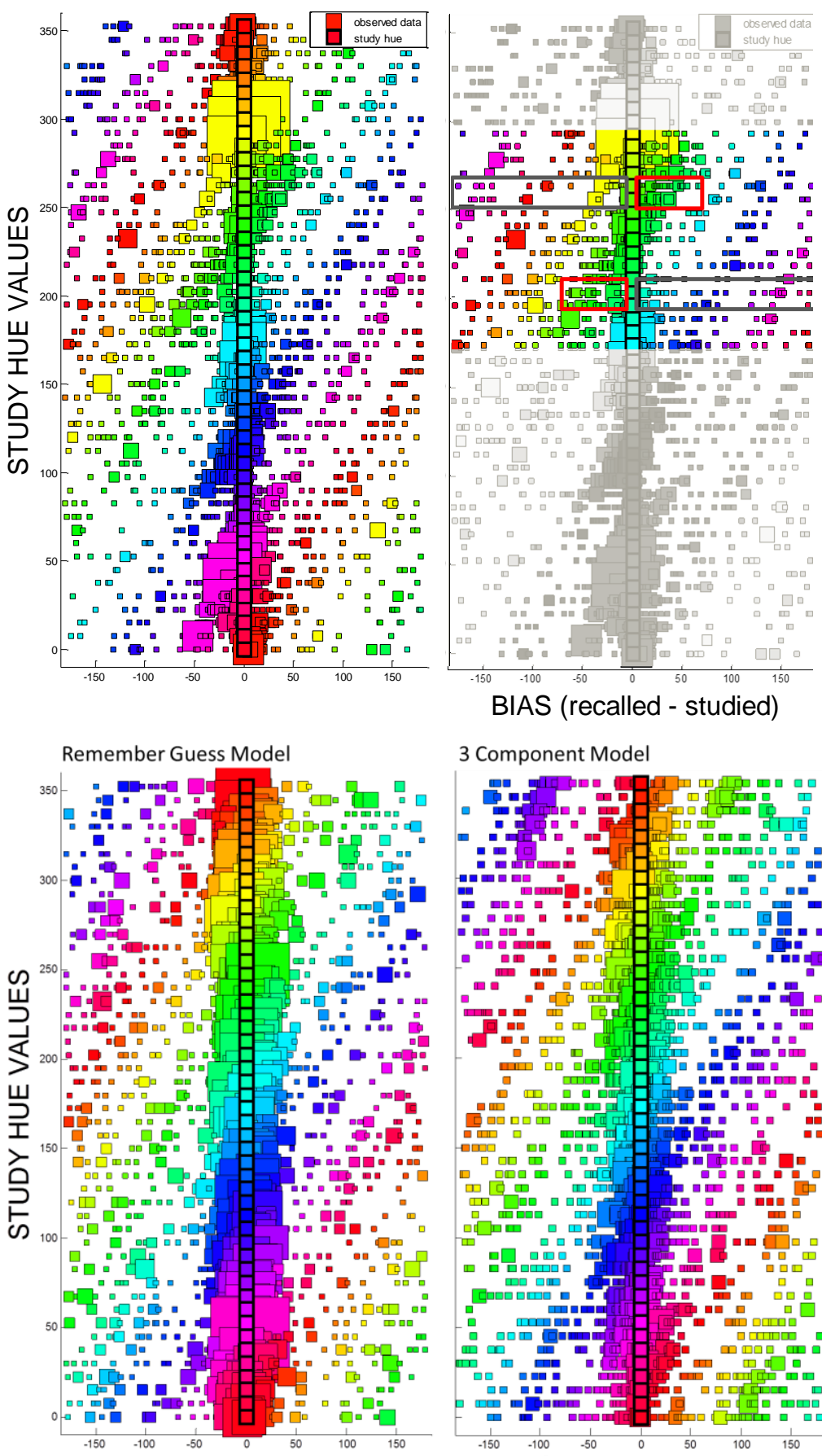

Regression fits

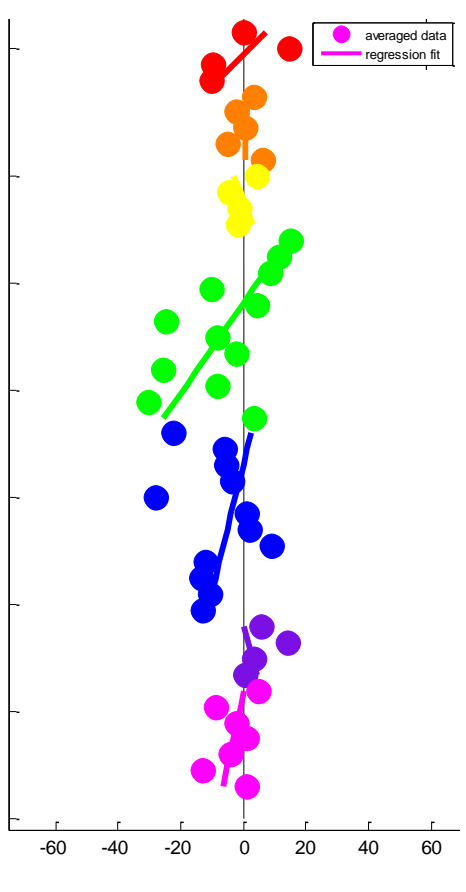

Bayesian Mixture Model

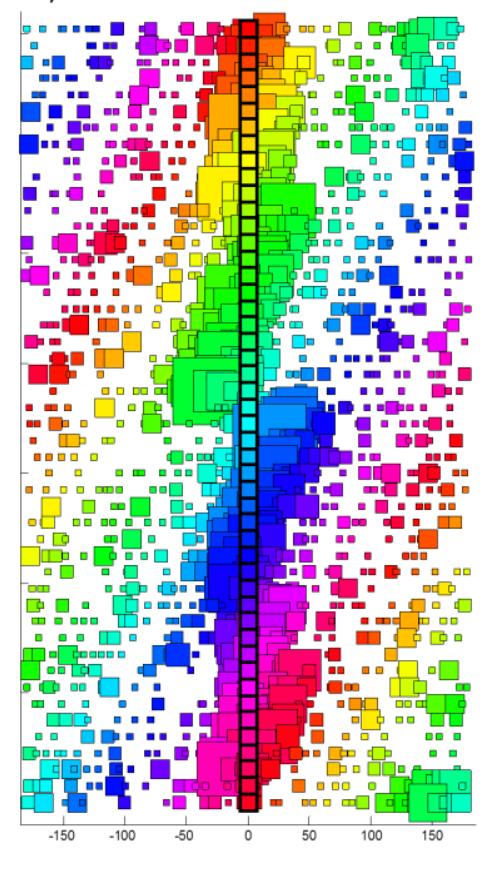

Figure 3. Recall response bias to studied hue values. Top left panel: All responses for a given study hue value appear in a horizontal row. The response markers are scaled by the frequency at which they were given (larger boxes indicate greater frequency) and colored with the exact hue value chosen. Top middle panel: Illustration of the asymmetry that results from a regression to the mean effect. Top right panel: Regression lines fitted to averaged recall data within 3 Tau of study values, by color category (see Persaud \& Hemmer, 2014 for category boundaries). Here red has been wrapped to show the regression line for the category. In other panels, red reflects its true location in color space, which is on both ends of the spectrum. Bottom row panels: simulated recall responses from the $\mathrm{RG}, 3 \mathrm{CRG}$, and $\mathrm{BM}$ models, respectively. 
Duncan, 1991; Huttenlocher, Hedges, \& Vevea 2000; Hemmer, Persaud, Kidd, \& Piantadosi, 2015).

2.2.3 Regression analysis Based on established universal categories (red, orange, yellow, green, blue, purple and pink; Berlin \& Kay, 1969), we assume that the observed recall bias is toward these seven categories (also, see Persaud \& Hemmer, 2014). A linear regression model was fitted to each subject for each category (Figure 3, top right panel). Because the regression effect is assumed to operate on memory (not guessing), the data were trimmed to remove responses assumed to be guessing. It is unclear prior to model fitting how to determine a guessing trial, therefore, data were trimmed following two different procedures: First, singletons in the data, grouped over response frequency, (Figure 3, top left panel) were removed and only responses within 75 hue values of the study value were considered for the analysis. A t-test of the subject slopes for each category found that slopes were significantly different from zero for all categories except orange, yellow and purple [red: $t(60)=-2.82, p<0.001$; orange $t(60)=-0.15$, $p=0.44$; yellow: $t(60)=1.96, p=0.97$; green: $t(60)=-3.82, p<0.001$; blue: $t(60)=-1.78, p=0.04$; purple: $t(60)=0.94, p=0.83$; pink: $t(60)=-1.65, p<0.05]$. Mean slopes were red: -0.69 ; orange: 0.02; yellow: 0.27; green: -0.47; blue: -0.17; purple: 0.2; pink: -0.16. Second, guessing responses were trimmed based on the inferred parameters from the Bayesian mixture model to all data. Thus, only responses within 3 standard deviations $[\tau=25.88]$ of the study value were considered for the analysis. A t-test of the subject slopes for each category found that slopes were significantly different from zero for green $[\mathrm{t}(60)=-5.09, \mathrm{p}=0.00]$ and blue $[\mathrm{t}(60)=-1.87, \mathrm{p}=0.03]$, marginally significant for red $[\mathrm{t}(60)=-1.38, \mathrm{p}=0.09]$, but not for orange, yellow, purple or pink. Mean slopes were red: -0.36; orange: 0.12; yellow: 0.54; green: -0.30; blue: -0.10; purple: 0.93; pink: -0.16 . 


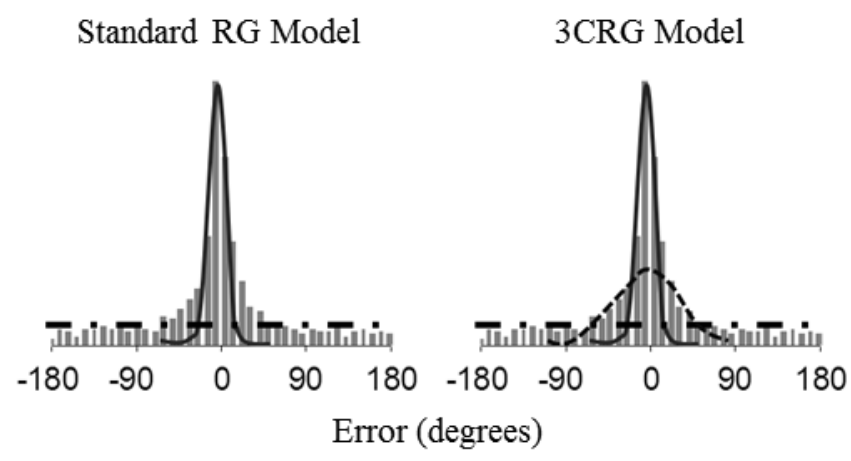

Figure 4. Model predictions. Left panel shows stylized predictions of the fit of the standard RG model to the aggregate data. The solid black line represents the memory contribution and the dashed line represents guessing. Right panel shows predictions of the fit of a 3CRG model to capture the 'shoulder' of the error distribution. The --dashed black line represents the contribution of the additional component in memory.

Visual inspection of Figure 3 suggests that the lack of significant regression in the purple category might be due to purple not being as salient as any of the other categories. Furthermore, the lack of significant regression in the yellow category might be due to yellow being the smallest category, and because of very high accuracy. Both orange and yellow also appear to have large overlap with the red category.

\section{Modeling}

To investigate the components of the error distributions and the observed regression patterns, we implemented two extensions of the standard remember-guess (RG) model: A three component remember-guess (3CRG) model, and a Bayesian mixture (BM) model. The standard RG model assumes that the error distributions are composed of two elements, a Gaussian-like memory distribution and a uniform distribution. See Figure 4, left panel for a graphical illustration of predictions for the RG model. Based on our observed pattern of data in the center portion of the error distribution, we predict that the combination of these two distributions will miss some of the area in the error distribution. If the memory component captures the peak of the 
error distribution, then it may miss the 'shoulders' and vice versa. This combination of a peak and shoulders might signal multiple components in memory.

To explain this pattern in the data, we first implement a simple extension assuming that memory is drawn from two normal distributions, one with high precision and one with lower precision. This additional parameter will allow the model to capture both the peak and the shoulder of the error distribution. See Figure 4, right panel for a graphical illustration of predictions for the 3CRG model.

Another pattern in the data that cannot be explained by the RG model, or by our first model extension, is the regression to the mean effect (we will return to a detailed regression analysis in the model comparison sections. See 4.3 Comparison of Regression). The 3CRG model also does not give a theoretical explanation of what the extra component (the low precision memory distribution) represents. Therefore, we implement a second extension-a Bayesian mixture model—which assumes that the additional component is the contribution of prior category knowledge. Rather than the mixture of two separate Gaussian distributions in the 3CRG model, the BM model assumes a single Gaussian distribution where the mean is a weighted linear combination of memory and prior knowledge. This model inherently predicts the regression to the mean effect. However, this effect is obscured in the error distributions, and necessitates the evaluation of the model to the full range of responses, rather than aggregate errors. Next, we detail the implementation of the three models and describe the results of the model comparisons.

\subsection{Standard 'Remember-Guess' Model (RG)}

We implemented the standard RG model using the MemToolbox (Suchow, Brady, Fougnie, \& Alvarez, 2013; memtoolbox.org). In this model, the probability density function is given by,

$$
(1-g) * \text { von } \operatorname{Mises}(0, \sigma)+g * \operatorname{Unif}(-180,180)
$$


Table 1. Model Parameter Values (confidence intervals)

\begin{tabular}{|c|c|c|c|c|}
\hline \multicolumn{3}{|c|}{ Remember-Guess Model (RG) } & & \\
\hline & $\begin{array}{c}\text { Fidelity (Conf. Int.) } \\
\sigma \sigma\left(^{\circ}\right)\end{array}$ & $\begin{array}{c}\text { Guess Rate(Conf. Int.) } \\
g\end{array}$ & & \\
\hline Lag 1 & $11.85(11.85-13.49)$ & $0.06(0.03-.010)$ & & \\
\hline Lag 2-3 & $16.15(14.40-18.25)$ & $0.42(0.37-0.46)$ & & \\
\hline Lag 4-9 & $17.63(15.82-19.70)$ & $0.49(0.46-0.53)$ & & \\
\hline Lag 10+ & $15.02(12.03-20.67)$ & $0.61(0.53-0.69)$ & & \\
\hline All & $15.82(15.03-17.13)$ & $0.46(0.44-0.48)$ & & \\
\hline \multicolumn{5}{|c|}{3 Component Remember-Guess Model (3CRG) } \\
\hline & $\begin{array}{c}\text { Fidelity (Conf. Int.) } \\
\sigma\left(\left(^{\circ}\right)\right.\end{array}$ & $\begin{array}{c}\text { Fidelity (Conf. Int.) } \\
\tau\left({ }^{\circ}\right)\end{array}$ & $\begin{array}{c}\text { Guess Rate(Conf. Int.) } \\
g\end{array}$ & $\begin{array}{c}\text { Mixing Parameter } \\
w^{*}\end{array}$ \\
\hline Lag 1 & $11.44(9.87-15.84)$ & $28.76(13.68-58.43)$ & $\mathbf{0 . 0 3}(0.01-.09)$ & 0.28 \\
\hline Lag 2-3 & $15.27(13.68-17.69)$ & $27.87(20.90-36.96)$ & $0.40(0.36-0.45)$ & 0.35 \\
\hline Lag 4-9 & $17.13(15.12-19.69)$ & $29.69(24.12-48.72)$ & $0.48(0.44-0.51)$ & 0.37 \\
\hline Lag 10+ & $15.21(12.44-32.11)$ & $35.21(13.36-73.98)$ & $0.59(0.51-0.68)$ & 0.30 \\
\hline All & $15.40(15.24-16.74)$ & $28.51(22.95-32.50)$ & $\mathbf{0 . 4 4}(0.42-0.47)$ & 0.35 \\
\hline \multicolumn{5}{|c|}{ Bayesian Mixture Model (BM) } \\
\hline & $\begin{array}{c}\text { Fidelity (Conf. Int.) } \\
\psi\left(\left(^{\circ}\right)\right. \\
\end{array}$ & $\begin{array}{c}\text { Fidelity (Conf. Int.) } \\
\tau\left(\left(^{\circ}\right)\right. \\
\end{array}$ & $\begin{array}{c}\text { Guess Rate(Conf. Int.) } \\
g \\
\end{array}$ & $\begin{array}{c}\text { Mixing Parameter } \\
w^{*}\end{array}$ \\
\hline Lag 1 & $22.12(17.22-23.85)$ & $23.43(14.10-26.00)$ & $\mathbf{0 . 0 5}(0.01-.09)$ & 0.49 \\
\hline Lag 2-3 & $19.80(14.17-19.80)$ & $21.12(17.40-22.09)$ & $\mathbf{0 . 4 3}(0.39-0.58)$ & 0.48 \\
\hline Lag 4-9 & $18.55(16.11-18.55)$ & $25.27(23.81-25.89)$ & $\mathbf{0 . 5 4}(0.54-0.62)$ & 0.42 \\
\hline Lag 10+ & $20.29(12.96-27.71)$ & $250.6(242.0-254.9)$ & $0.60(0.51-0.72)$ & 0.08 \\
\hline All & $19.03(18.77-20.70)$ & $25.88(23.68-26.79)$ & $0.47(0.47-0.52)$ & 0.42 \\
\hline
\end{tabular}

where remembered responses are von Mises distributed (due to the circular hue space) with a mean of $\mu$ and standard deviation $\sigma$. Guessing responses are produced with probability $g$ and are uniformly distributed across the stimulus range from -180 to 180 degrees. Furthermore, because the error distribution is centered on zero $\mu=0$, this parameter will not be considered in this implementation.

Table 1 gives the inferred parameters and $95 \%$ confidence intervals. Figure 3, bottom left panel shows the simulated draws from the posterior of the RG model. According to the model fits, there is a substantial increase in memory noise $(\sigma)$-i.e., decrease in memory fidelitybetween lag 1 and lags 2-3. Thereafter, memory fidelity appears relatively constant (overlap in 
confidence intervals between lag groupings). In addition, there is a steady increase in the guessing rate $(g)$ from lag 1 and forward. The model appears to capture the general trend in the data, with the exception of missing the peak of the distribution at some lags and a small portion of the shoulder at others.

\subsection{Three component 'Remember-Guess' Model (3CRG)}

Next, we implement the first extension. We assume that the memory component is itself a mixture of two Gaussian distributions. This is very similar to the Donkin et al. (2014) model, which assumes two components in guessing, where the extra component only applies at retrieval. Our model, in contrast, makes the assumption that the increased noise is attached to the memory component rather than the guessing component. Our memory mixture is also not conditioned on labeling, but rather applies to all trials. While mathematically the two models are equivalent, they differ in the conceptual underpinnings.

In the 3CRG model, we first assume the additional component is related to the memory component in anticipation of the BM model. Second, we use the noise from the two Gaussian components to determine the mixing, rather than assume an additional free parameter. Third, the assumption that the additional component attaches to memory is agnostic about whether the influence of the additional component happens at encoding or retrieval.

The probability density function of the $3 \mathrm{CRG}$ model is then given by,

$$
\begin{gathered}
(1-g) *\left((1-w) * \text { von } \operatorname{Mises}\left(0, \sigma_{\text {mem }}\right)+w * \operatorname{von} \operatorname{Mises}(0, \tau)\right)+\operatorname{Eq}(2) \\
g * \operatorname{Unif}(-180,180)
\end{gathered}
$$

We assume that the mixture of von Mises distributions $w$ is based on the fidelity of these two distributions. This is strongly motivated by the assumption of the BM model that the linear weighting is a Bayesian integration such that $w=\left(1 / \tau^{2}\right) /\left[\left(1 / \tau^{2}\right)+\left(1 / \psi^{2}\right)\right]$. For clarity this can be 
rewritten as $w=\psi^{2} /\left[\tau^{2}+\psi^{2}\right]$. Using the noise parameters in this way ensures a tradeoff between the two memory components such that when one has high precision it carries more weight, which seems a reasonable assumption of memory. Furthermore, the noise in one of the von Mises distributions is dependent on the noise in the second distribution, $\sigma_{\mathrm{mem}}=\sqrt{ }\left(1 /\left[\left(1 / \tau^{2}\right)+\right.\right.$ $\left.\left(1 / \psi^{2}\right)\right]$. Using the noise parameters in this way establishes a difference in the precision on the two von Mises distributions, such that $\sigma_{\mathrm{mem}}$ is always smaller than $\tau$, and that when $\psi$ and $\tau$ are the same, the noise on one von Mises is smaller than the other ${ }^{1}$.

It should be noted that for completeness, we also implemented a number of other variations of the 3CRG model including versions where: 1) the weighting $w$ is inferred but $\sigma_{\text {mem }}$ is still calculated from $\psi$ and $\tau$ as above, 2) the weighting $w$ is calculated as above, but $\sigma_{\text {mem }}$ as the noise on one von Mises is replaced with $\psi$, treating the noise parameters as independent (See Appendix A4), and 3) all parameters are inferred, that is the weighting $w$ is inferred, $\psi$ is the memory noise on one von Mises and $\tau$ as noise on the other-that is, $\sigma_{\mathrm{mem}}$ is not calculated as above (See Appendix A5). Alternate version 1) proved to be very unstable at lag $10+$ in the hierarchical fitting (see section 4.2 for hierarchical fitting of the RG, 3CRG and BM models), and we ultimately abandoned this model. Alternate version 2) provided identical patterns in parameter values as the version implemented in Eq. 2 above, identical values of AIC and BIC, and an even better DIC score. However, the consequence of not tying the noise parameter via $\sigma_{\text {mem }}$ means the model is agnostic about which component is the primary and which is the secondary, and for lag 10+ the hierarchical fitting would sometimes switch whether $\psi$ had the smaller value or $\tau$ had the smaller value. Alternate version 2) was also unstable in the hierarchical fitting, but adds some interesting insights (See Appendix A5 for discussion). It

\footnotetext{
${ }^{1}$ As a toy example, if $\psi=20$ and $\tau=50, \sigma_{\mathrm{mem}}=18.6$ If $\psi=20$ and $\tau=30, \sigma_{\mathrm{mem}}=16.66$. If $\psi=20$ and $\tau=20, \sigma_{\mathrm{mem}}=14.1$
} 
should also be noted that the 3CRG model (in any of these versions) is very stable when fitted at individual lags, indicating the robustness of the model.

Table 1 gives the inferred parameters of the 3CRG model. Figure 3, bottom middle panel shows the simulated draws from the posterior of the 3CRG model. Similar to the model fits of the standard RG model, there is an increase in memory noise $(\sigma)$-i.e., decrease in fidelitybetween lag 1 and lags 2-3, and memory noise stabilizes across remaining lags. The second memory noise parameter $(\tau)$ follows a similar trajectory. There is also an increase in the guessing rate $(g)$ from lag 1 and forward. Overall, the 3CRG model posits a similar noise in memory for the $\sigma$ parameter, and a fairly similar guessing rate, relative to the standard RG model. In this respect, our findings are remarkably consistent with Zhang and Luck (2008), Brady et al. (2013), and Donkin et al. (2014). The failure of the standard RG model to capture the shoulder of the central error distribution is accounted for by the additional noise parameter of the 3CRG model, while simultaneously providing a better fit to the peak of the distributions (for model comparison see Table 2).

\subsection{Bayesian Mixture Model (BM)}

Motivated by the experimental results, which show a regression to the mean (see Figure 3, top right panel) for a number of color categories, we sought to develop a model that could take into account this behavior. We propose a Bayesian mixture model where recall is a combination of three inputs: noisy representations stored in memory, prior expectations (category knowledge), and random guessing. This approach combines the likelihood from the Bayesian Cognitive model (BCM) developed by Hemmer and Steyvers (2009b) and Hemmer, Tauber, and Steyvers (2015) with the standard RG model of Zhang and Luck (2008). Importantly, in order to 
visualize the full range of samples from the posterior to demonstrate the regression to the mean effect, we now fit the model to the observed responses, rather than the error distributions. ${ }^{2}$

We extend the RG model by assuming that responses are based on a combination of samples drawn from memory, with probability $w$, and prior expectations, and otherwise, with probability $g$, responses are assumed to be guesses. In the BM model, standard Bayesian techniques

(Gelman et al., 2003) can be used to compute the mean of the posterior distribution:

$$
\text { Recall } \sim \mathrm{N}\left((1-w) * \bar{y}+w * \mu, \sigma_{\mathrm{mem}}\right)
$$

where recall is a weighted linear combination, of samples $y$ drawn from memory with noise $\psi$ and some prior expectation with mean $\mu$ and standard deviation $\tau$, for the stimulus feature, and with fidelity $\sigma_{\text {mem }}=\sqrt{ }\left(1 /\left[\left(1 / \tau^{2}\right)+\left(1 / \psi^{2}\right)\right]\right.$. The $\mu$ for each category was specified based on the assessment of expectations for color categories in Persaud and Hemmer (2014; See Persaud \& Hemmer, 2014 for predictions from the Bayesian model over the true color space). ${ }^{3}$ The weights are a combination of the noise in memory and the fidelity of the prior, such that $w=\left(1 / \tau^{2}\right) /\left[\left(1 / \tau^{2}\right)\right.$ $\left.+\left(1 / \psi^{2}\right)\right]$. The probability density of recall is given by

$$
\begin{gathered}
(1-g) * \operatorname{von} \operatorname{Mises}\left((1-w) \bar{y}+w \mu, \sigma_{\mathrm{mem}}\right)+ \\
g * \operatorname{Unif}(0,360)
\end{gathered}
$$

Table 1 gives the inferred model parameters for the BM model. Different from the model fits of both the standard RG model and the 3CRG model, there is no change in memory noise ( $\psi)$ between lag 1 and lags 2-3, rather memory noise is stable across all lags. The noise on the prior $(\tau)$ grows slightly from lag 2-3 to lag 4-9 and then jumps dramatically for lag $10+$. The weighting $w$ is steady and evenly split between the memory trace and the prior until lag $10+$

\footnotetext{
${ }^{2}$ We also refitted the standard remember-guess model to the full response distribution. See Appendix Table A3. There is no difference in the parameters of this model between the two fittings.

${ }^{3}$ Category means: $1.65^{\circ}, 30.35^{\circ}, 60.08^{\circ}, 119.69^{\circ}, 230.30^{\circ}, 284.12^{\circ}, 323.40^{\circ}$
} 
where, in response to the large increase in $\tau$, it decreases. As in the RG and 3CRG models the guessing rate $(g)$ increases gradually from lag 1 and forward.

Figure 3, bottom right panel shows the simulated draws from the posterior of the BM model. Both the RG model and 3CRG model simulations (Figure 3, bottom left and middle panels) show a mass of responses near the center zero-bias line and a uniform spread of remaining responses to either side. That is, responses are equally likely to be over and under-estimated regardless of the study hue value relative to the mean of the color categories. See section 4.3 for regression fit to the RG and 3CRG models. Unlike the RG model and 3CRG model, the BM model can capture the regression to the mean effect, where simulated responses for hue values greater than the category means are more likely to be underestimated, while values less than the mean are more likely to be overestimated, creating an asymmetry similar to the raw data.

\section{Model Comparison}

\subsection{Comparison by lag}

Model comparison between the RG and the 3CRG models was conducted using the MemToolBox (Suchow, et al, 2013; memtoolbox.org). The AIC and BIC values for the two

Table 2. AIC and BIC Model Comparisons by Lag Group

\begin{tabular}{lcccccc}
\hline & \multicolumn{3}{c}{ AIC } & & \multicolumn{3}{c}{ BIC } \\
\hline & RG & 3CRG & BM & RG & 3CRG & BM \\
\hline Lag1 & 1663.16 & $\mathbf{1 6 5 7 . 4 7}$ & 1915.22 & 1673.42 & 1672.87 & 1930.62 \\
Lag 2-3 & 9264.21 & $\mathbf{9 2 4 9 . 7 6}$ & 9647.52 & 9277.43 & $\mathbf{9 2 6 9 . 5 9}$ & 9667.35 \\
Lag 4-9 & 17090.03 & $\mathbf{1 7 0 7 4 . 1 4}$ & 17477.20 & 17104.41 & $\mathbf{1 7 0 9 5 . 7 2}$ & 17498.77 \\
Lag 10+ & 3182.68 & 3182.96 & 3232.12 & $\mathbf{3 1 9 3 . 4 5}$ & 3199.43 & 3248.58 \\
All data & 31374.58 & $\mathbf{3 1 3 3 2 . 2 2}$ & 32275.59 & 31390.22 & $\mathbf{3 1 3 5 5 . 6 7}$ & 32298.79 \\
\hline
\end{tabular}

*Bold font indicates better fits with a difference score greater than 5, while italicized font indicates marginally better fits with a difference score less than 5 . 
models are reported in Table 2 (bold font indicates better fits with a difference score greater than 5, while italicized font indicates marginally better fits with a difference score less than 5). Due to the fact that each participant only performed 48 trials with varying lags leading to a sparsity of data for some lags, individual differences were not assessed. The data was pooled across subjects, and subjects were treated as fixed in both AIC and BIC. An improved fit was observed for the 3CRG model for the aggregate error distribution and all lag groupings, except 10+. It seems reasonable that the model comparison favors the standard RG model at lag 10+ given the increase in the guessing component of the error distribution. The uniform distribution has lifted and could potentially account for the portion of the error distribution that would be accounted for by the second fidelity component parameter. While the improvement is marginal for lag 1 (less than a 5 point difference in AIC between RG and 3CRG), the improvement is substantial for lags 2-3 and 4-9. Memory at lag 1 appears to have a high level of precision and a majority of the performance can be attributed to remembering with little influence of guessing. In contrast, memory is both precise and less precise at other lags and there is a greater rate of guessing. This is consistent with the $3 \mathrm{CRG}$ assumption that there is both a memory component with high fidelity and a component with greater noise. The 3CRG model makes it clear that there is additional information in the error distribution that cannot be solely explained by remembering with noise and guessing (i.e. the RG model).

The AIC and BIC values for the BM model are also reported in Table 2. No improvement in fit was observed for the BM model relative to either the RG or the $3 \mathrm{CRG}$ models. There are several reasons why the BM model might lose out in the model comparison. For example, we assume only one value for tau for all categories, and we specify the color categories based on universal color categories. Furthermore, the weak regression effects in the data allow the 3CRG 
model to successfully fit all the data without accounting for the regression effects. We discuss all of these reasons along with possible remedies in the discussion section. It is important to note that making allowances for an influence of category information in the BM model produced the characteristic regression to the mean effect which cannot be captured by the two other models, and we still see this as a substantial strength of the BM model. Restricting analysis to error models — while producing an improved fit—leads to very different conclusions about memory. The regression effect makes it clear that category knowledge plays an important role in recall, and that this must be considered in models of LT memory.

\subsection{Hierarchical model comparison}

Thus far, we have evaluated the models based on fits at the individual lags. It is reasonable, however, to assume that the same model applies to all lags. Therefore, in addition to fitting the separate models for each lag, we also fitted a single hierarchical model to all lag groupings together, for each of the three models. This model treats each of the lag groupings parameters as samples from a normally-distributed population and then infers both best fitting parameters for each lag grouping, as well as the population mean parameter.

Because AIC and BIC are not appropriate for assessing hierarchical models, here we report DIC scores (Deviance Information Criterion; Spiegelhalter et al., 2002, van der Linde, 2005). The DIC is a generalization of the AIC for hierarchical models, which penalizes both for quality of fit and number of parameters. As before, the fitting was conducted using the MemToolBox.

The parameters for each of the three hierarchical implementations were essentially identical to the parameters reported in Table 1 across all lag groupings. However, due to the sparsity in the data at lag $10+$ some of the models are very sensitive to the choice of prior distribution. This particularly affects the BM model in the hierarchical implementation. The DIC for the models 
were as follows: $\mathrm{RG}=31280,3 \mathrm{CRG}=31230$, and $\mathrm{BM}=32173$. This replicates the pattern of model comparison when lags are estimated separately. There is an improved fit observed for the 3CRG model over both the RG and BM models.

\subsection{Regression Comparison}

To further understand how the models capture the observed data, a regression analysis was performed on the simulations from each of the three models, similar to the regression analysis performed on the subject data (see section 2.2.3). We simulated draws from the RG model assuming 61 subjects and 48 study hue values as in the experiment (Figure 3, bottom left panel). Because the regression effect is assumed to operate on memory (not guessing), only responses assumed to be drawn from memory (within 3 standard deviations [ $\sigma=15.82]$ of the study value) were considered for the analysis. A linear regression model was fitted to each simulated subject for each of seven universal color categories: red, orange, yellow, green, blue, purple and pink (similar to the regression analysis for the raw data). Recall that, t-tests of the observed subject data revealed that the slopes of 4 of the 7 categories were significantly different from zero. In stark contrast to the subject data, one sample t-tests of the RG model slopes failed to find a significant difference from zero in any category. The mean slopes for all categories were: red: -0.04; orange: -0.16; yellow: -0.31; green: 0.09; blue: 0.11; purple: 0.07; pink: -0.05 .

We simulated draws from the 3CRG model following the same procedure as for the RG model. Responses within 3 standard deviations $[\tau=28.51]$ of the study value were analyzed. Onesample t-tests of the 3CRG model slopes failed to find a significant difference from zero in all categories, except purple $[t(60)=-1.90, p=0.03]$. Note that the observed subject slopes are not significantly different from zero for the purple category, and thus, the 3CRG model does not 
mirror the subject data for this category. Mean slopes were red: 0.79 ; orange: -0.62 ; yellow: 0.29; green:-0.07; blue: -0.04; purple: -0.98; pink: -0.29.

Lastly, we simulated draws from the BM model following the same procedure as for the other models. Responses within 3 standard deviations $[\tau=25.88]$ of the study value were analyzed. One sample t-tests for the simulated BM model data revealed a similar pattern to the subject data, in that 4 of the category slopes were different from zero (yellow: $t(60)=-4.26, p=.00$; green: $t(60)=-3.62, p=.00$; blue: $t(60)=-4.18, p=.00$; pink: $t(60)=-3.68, p=.00)$. The mean slopes for all categories were red: -0.48; orange: -1.07; yellow: -0.17; green:-0.36; blue: -0.37; purple: 0.30; pink: -0.65 .

For completeness, we then compared the slopes from the subject data for each category to the slopes of the simulated data. We sought to evaluate whether observed regression patterns in the subject data were observed in the model simulations - i.e., in the categories in the subject data where the slopes were significantly different from zero, the model simulations also resulted in non-zero slopes of the same degree. For the RG model, there were significant and marginal differences in slopes, when compared to the subjective slopes, for four categories (red: $t(120)=$ 1.74, $p=0.08$; yellow: $t(120)=2.12, p=0.04$; green: $t(120)=-2.87, p=0.00$; blue: $t(120)=-2.38$, $p=0.02$ ). This was due to the $\mathrm{RG}$ model either failing to predict a regression (red and green), or predicting an effect in the opposite direction of the observed data (yellow and blue). In the three remaining categories, the failure to find significant differences between the model and the subject data was due to the RG model predicting no regression when there was no regression effect in the observed data (orange and purple), or when the regression effect in the data was weak (pink). In total, the RG model only correctly predicted two categories-yellow and purple. 
Similar to the RG model, for the 3CRG model there were significant and marginal differences in slopes, when compared to the subjective slopes, for four categories (red: $t(120)=-2.24, p=$ 0.03; yellow: $t(120)=1.72, p=0.09$; green: $t(120)=-2.53, p=0.01$; purple: $t(120)=2.11, p=0.04)$. This was due to the $3 \mathrm{CRG}$ model either failing to predict a regression (green), predicting the effect in the opposite direction (red and yellow), or predicting a strong regression when there was no observed regression in the data (purple). In the remaining three categories, the failure to find significant differences was due to the $3 \mathrm{CRG}$ model predicting a weak, but non-significant regression effect, when there was a weak, but significant effect in the data (blue and pink), or predicting a weak, but marginally significant regression, when there was no regression effect in the data (orange). In total, the 3CRG model only correctly predicted the regression pattern in the blue and pink categories.

In contrast, for the BM model, there was no significant difference in five of the seven categories. This means that the BM model either predicted a regression to the category mean (red, green, and blue) or no regression (yellow and purple) for the same categories as was observed in the data. In one category (pink: $t(120)=2.47, p=0.02$ ), the observed difference is due to the BM model over-predicting the steepness of the regression, rather than failing to predict the regression effect. Only in one category (orange: $t(120)=3.59, p=0.00$ ), does the BM model fail to predict the pattern in the subjective data - by predicting a negative slope when the slope in the subject data, although negative, was not significantly different from zero.

In summation, the collective results of the regression analyses suggests that the slopes generated from the BM model more closely resemble the regression behavior in the subject data, compared to both the RG and 3CRG models.

\section{Discussion}




\subsection{Summary}

We investigated the time course of errors in recall in an effort to understand the components that contribute to LT episodic memory. We employed a novel experimental paradigm and conducted a lag analysis to characterize the influence of category knowledge, and memory over time. We then implemented three distinct cognitive models to evaluate the potential contributing components to memory. Furthermore, we found that there are two important factors in LTM that cannot be accounted for by the standard RG model. In the aggregate, recall reflects a combination of three components: a peaked memory component, a less precise memory component, and a guessing component, capturing the peak and 'shoulders' in the error distributions. In the full response data, recall reflects regression to the mean effects for several color categories, indicating a contribution of prior category knowledge to memory.

The 3CRG model can account for the additional component in memory, and provided a large improvement in the fit over the RG model. The benefit of the 3CRG model is that it has an additional component that can account for a number of mechanisms that might influence LT memory, such as verbal labeling (Donkin et al., 2014), and variable precision in memory (van den Berg et al., 2012). Despite the strengths of the 3CRG model, there is no clear theoretical interpretation of what is encompassed in this component. Moreover, like the RG model, it also cannot capture the regression patterns in the data. The BM model, in contrast, can account for both a second memory component and the regression patterns, and the BM model also has a theoretical framework for the additional component. It, however, loses dramatically in the model comparisons. The BM model we implemented here is a first pass at understanding the influence of category knowledge, and there are a number of factors that might account for the $3 \mathrm{CRG}$ model being favored over the BM model, such as weak regression effects in the data, fragile 
associations, incorrect category assumptions and other general modeling assumptions. There are also several possible remedies that might improve the BM model and are discussed in the next section. Furthermore, our results have important implications for understanding mechanisms such as decay, sudden death and interference.

\subsection{Weak regression effects}

A key assumption of the BM is the regression to the mean effect. This effect has been demonstrated to be robust in memory (Hemmer \& Persaud, 2014; Hemmer \& Steyvers, 2009a; Hemmer, Tauber, Steyvers, 2015; Huttenlocher, Hedges, and Duncan, 1991; Huttenlocher, Hedges, \& Vevea 2000; Persaud \& Hemmer, 2014; Hemmer, Persaud, Kidd, \& Piantadosi, 2015). In our data however, using seven universal color categories as a benchmark resulted in poor alignment to the data. The regression analysis revealed that there was no significant regression in three categories, suggesting that the use of universal color categories in the regression assumptions is likely not representative of our data. Furthermore, the fact that the 3CRG model outperforms the BM model, in both AIC and BIC, suggests that the regression effects are weak enough that the inability of the $3 \mathrm{CRG}$ model to fit the regression effects is outweighed by its improved fit to the rest of the data. While Persaud and Hemmer (2014) found strong regression effects to all seven universal color categories, they conditioned their regression analysis on responses where participants also provided the correct verbal label for the study value at test. In other words, they only analyzed data where the participants were able to recall the association between the test cue (shape) and the study color. Here we include all data, which likely includes trials where participants misassociated shape cues to studied colors, guessed, or made some other error. A key test for the flexibility of 3CRG model without accounting for regression effects would be if the model still outperformed the BM model for the finding of 
differential bias to two separate categories for stimuli studied at the same size (i.e., a large strawberry and a small apple-See Hemmer \& Steyvers, 2009a).

We acknowledge that our findings are likely data dependent. There are several possible considerations that might improve the fit of the BM model, or help to lend further support for the strength and flexibility of the 3CRG model. Since the stimuli were drawn from the true hue space, categories had varying sizes. An example of this can be seen in Figure 3, top left panel, where the raw data shows high accuracy (large squares) around the yellow category, because this is a very small category. A possible future extension to the BM model would be one that considers variable precision in the Tau parameter (here we have assumed that there is only one value of tau for all categories). This would be akin to the van den Berg et al. (2012) variableprecision model which assumes variability in the precision with which items are encoded, but with variable precision in the categories. This could remedy the weak regression effects in small categories which obscures the importance of capturing the regression effects in other categories.

\subsection{Alternative color categories}

The samples drawn from the posterior of the BM model (Figure 3 bottom right) reveals a misalignment between the color categories used to inform the model, and the actual categories borne out in the data. For example, in the data there appears to be two blue categories-light blue and dark blue. However, the BM model only exhibits regression to one blue category -consistent with universal categories. To better understand what color categories participants might have regressed toward in the response data, we conducted a cluster analysis (see Appendix A6). Interpreting the clusters relative to the standard universal color categories, suggests that observers may be using eight categories - five of which can be interpreted relative to the universal color categories: a category composed of red, orange, and yellow universal color 
values (visualized in red; Figure A1); another category predominately composed of green values; two separate categories for the hue space encompassing blue values (visualized in light blue and dark blue); one category for purple; and one for pink (although pink may contain red values, given the circular nature of the hue space). Interestingly, there were two uniform clusters that span the entire hue range and fell on the top and bottom edges of the graph. These clusters may potentially correspond to the guessing component, or could relate to the large value for $\tau$ at lag $10+$ in the BM model. Participants also appear to use color categories at various levels in the color hierarchy. For example, participants appear to use the subordinate categories of light blue and dark blue. On the other hand, for colors in the universal red and orange ranges, they use a superordinate color category for warm colors (i.e. a blended category for red, orange, and yellow).

Another interesting feature of the cluster analysis is the natural prediction of regression to the mean behavior in the data. The inherent regression effect learned from the cluster analysis and the use of color categories with different boundaries, provide important constraints for future considerations of Bayesian modeling of color space. While we think that the BM model provides an important theoretical framework in considering regression effects and category influences, continued failures of the BM model even under improved category assumptions would lend further strength to the $3 \mathrm{CRG}$ model.

\subsection{Fragile associations}

Another factor that might impact the performance of the models - particularly in the individual lag fits - is that of fragile associations. Modeling paradigms in visual short-term memory have successfully extended the RG model to incorporate task-based components, such 
as "misassociation" or "misbinding” parameters (Bays, Catalao, \& Husain, 2009; Bays, Wu, \& Husain, 2011). There are some hints that there might be fragile associations in our data as well. At lag 10+, precision in the additional component in both the $3 \mathrm{CRG}$ and BM models is low and the rate of guessing is high, favoring the RG model. In fact, lag 10+ is the only lag grouping where the 3CRG model loses. This however, might be a consequence of the experimental design. Following standard procedures in color memory paradigms in visual working memory, we deliberately use an experimental design where we assign colors to random objects (e.g., Brady et al., 2013). An important consequence of this design, in conjunction with long lags, in the study of LTM, is that the object color pairing might be what is forgotten. In other words, performance at lag 10+ gives the appearance of a high rate of guessing, not because of a failure to remember the studied hues, but due to a failure of the shape cue to retrieve the correct hue pairing. A natural task with a stronger cue-target association might result in a substantially different pattern of data - one where the rate of guessing is lower. Recent work by Lew, Pashler, and Vul (2015) proposes an interesting new model of fragile associations in LTM. While this is beyond the scope of this paper, given that we cannot assess fragile associations in the current experimental paradigm, we agree that this is an important future direction. Fragile associations might hamper the BM model more than the other models because the behavior looks like guessing, but it has a strong memory trace, albeit bound to the wrong cue. Therefore, the model has a difficult time assigning the behavior, and the role of prior knowledge appears more diffuse.

\subsection{Interference vs. Decay}

Models of memory have varied in their mechanisms of forgetting. Some models theorize that forgetting occurs as a function of decay of memory traces over time (e.g., Barrouillet, Bernardin, \& Camos, 2004; Portrat, Barrouillet, \& Camos, 2008), while others attribute forgetting to 
interference (e.g., Lewandosky, Oberauer, Brown, 2009; Neath \& Brown, 2012). Our findings appear to provide support for both forgetting mechanisms. First, our results reveal a decrease in memory fidelity (increased noise in the models' $\sigma$ parameter) from lag 1 to lag 2-3 in the RG model, but in all three models memory fidelity then remains stable across remaining lag groups. This suggests that the memory trace initially suffers some decay during virtually shortterm/working memory, which supports the decay account (Baddeley \& Scott, $1971^{4}$ ), but is stable into LT memory. This progression in parameters also suggest that - although we are modeling the lag groupings under the assumption that one model should account for all groupings - there is something different about the data at lag 1 - namely very high precision, no second component and virtually no guessing, consistent with short-term/working memory.

While memory noise stays steady across lags, guessing $(g)$ increases across lags for all models. In this respect, our findings are remarkably consistent with Zhang and Luck, 2008, Brady et al., 2013, and Donkin et al., 2014. This has led to the interpretation that there is an upper bound on memory noise in LTM, and that memory suffers a 'sudden death' (Brady et al., 2013). Brady et al., 2013, however, could only make this assertion evaluating the transition from working to LT memory. Our design allows us to understand what happens across lags (time) in LTM. For the BM model, the noise in the prior $(\tau)$, exhibits a very different pattern from the RG model: $\tau$ is steady on lags $1-3$, then increases slightly for lags 4-9, but increases dramatically for lags $10+($ a similar pattern can be seen in the alternative implementation of the 3CRG model (Appendix A5) with all parameters inferred). As a result, the weighting ( $w$ ) of samples from memory and the prior changes across lags. This can be understood as sampling from different granularities of prior knowledge, consistent with hierarchical influences in LTM (e.g., Hemmer

\footnotetext{
${ }^{4}$ Although more recent work suggests that forgetting in short-term memory can also be explained by an interference account of forgetting (see Lewandosky, Oberauer, Brown, 2009 for other interference based views accounting for data traditionally thought to support the trace decay account).
} 
\& Steyvers, 2009), and the hierarchical nature of colors (Persaud \& Hemmer, 2014). On earlier lags One might use a specific prior (e.g., light red or dark red), but on intermediate lags One might use a prior of 'red', and at later lags, where the noise on the prior is very large, One might simply use a prior of warm versus cool—or some similar strategy. This progression in parameters in the BM model is contrary to the idea of sudden death. Taken together, our data suggests that not only is fidelity fixed in LTM, but also, category information plays an important role before One resorts to random guessing. Moreover, there is no decay in LTM and no sudden death.

This leaves interference (Neath \& Brown, 2012) as the likely mechanism for increased guessing; especially since the trials in our task are interleaved, and the target-cue bindings (color-shape pairing) are arbitrary in nature. Thus, by lag $10+$ it is possible that the memory trace (color) is present, but the association to the cue is difficult to retrieve as a result of studying other target-cue combinations. Such an interference explanation is consistent with a fragile association account of memory (Lew, Pashler, \& Vul, 2015), where recall is thought to be a combination of remembered information, misassociated information (incorrectly binding targets to cues), and guessing.

Recent work assessing event-based memory in rhesus monkeys lends further credence to interference being the mechanism of forgetting (Devkar \& Wright, 2016). Memory accuracy was found to decrease as a function of proactive interference, such that, previously presented stimuli (as far back as 16 trials) interfered with same/different recognition responses. Also, the influence of proactive interference did not change as a function of presentation time between study and test, and inter-trial time. In other words, longer delays between study and test and between trials, where previously studied information would have decayed, did not hamper interference (again, 
even when the information was studied 16 trials prior).

Serial dependencies are potentially another source of interference that appears as guessing. Serial dependences refer to the bias in memory that results from information experienced on previous and present trials. It has been demonstrated in visual perception that memory for one item is influenced by accompanying (even task-irrelevant) information and a running average over previous trials (Huang \& Sekuler, 2010). Similarly, the perceptual system is serial dependent in that perception is informed by both prior and present information (Fischer and Whitney, 2014).

While serial dependencies may be present at later lags before participants resort to guessing randomly, they are not the source of interference at earlier lags where category information is still available. Hemmer and Steyvers (2009a) showed that in LT memory, the regression to the mean effect is not a result of sequential dependencies. They demonstrated a differential bias when two items from different object priors (e.g., an apple and a strawberry) were studied at the same size. This is also the case in the data presented here (see Figure 3, top row middle panel) where there is a differential bias, for example on the boundary between yellow and green, where neighboring hue value results in regression to opposite categories. Sequential dependencies would result in an equal bias towards either category on the boundary dependent on the previous trial (i.e., if previous trial was green bias would be to green but if previous trial was yellow bias would towards yellow). A critically explicit prediction of the BM model is exactly the differential regression at category boundaries as observed in our data.

Given the design of the paradigm used in this investigation, we draw our conclusions with some caution. It is difficult to disentangle the roles of memory decay and interference as mechanisms of errors and forgetting because we do not control for rehearsal (Lewandosky, 
Oberauer, Brown, 2009; Portrat, Barrouillet, \& Camos, 2008), and trials are interleaved—which could result in intra-sequence interference (Neath \& Brown, 2012) - both factors that are required to discern between decay and interference. The decay versus interference differentiation is further complicated by the idea of equivalence, which suggests that both decay and non-decay models provide strong fits to the same data (Neath \& Brown, 2012).

Lastly, a contributing factor to memory fidelity, that is not explored in this work but is noteworthy, is the role of intentional forgetting. When participants are instructed to forget certain information in the study stimuli, this leads to a decrease in the probability that the memory trace is retrievable and a decrease in the overall fidelity of the memory trace (Fawcett, Lawrence, \& Taylor, 2016). In this way, memory intentions influence the quantity of information encoded into LTM and the quality of the information. Fawcett, Lawrence, \& Taylor (2016) modeled this finding using a hierarchical variable-precision mixture model similar to the standard RG model, with the allowance of variability in encoding similar to van den Berg et al (2012).

\subsection{Conclusions}

The implications of the findings from these three models highlight significant characteristics of LT memory. First, consistent with Donkin et al. (2014), there is a clear intermediate stage in LT memory between precise recall and random guessing. While the difference between our 3CRG model and the Donkin et al. (2014) model is a question of technical assumptions, the difference of these two models to the BM model, however, is one of core assumptions, namely that there is an influence of prior knowledge and a regression to known categories. We further argue that at this intervening step, there is a more generalized influence of expectations beyond verbal labeling. Notably, restricting analysis to error models that mask intervening steps leads to very different conclusions about memory. Deriving conclusions about memory based solely on 
error distributions is misleading in that it can obscure critical features of memory, such as the influence of prior category knowledge. Therefore, it is important that future research seeks to move beyond the standard remember-guess paradigm for LT memory, and work to elucidate the role of fragile associations and interference. We believe that we have clearly demonstrated that the 3CRG model is robust and consistently outperforms the other models, and that the BM model explains important patterns in the data.

\section{Acknowledgments}

This work is based upon work supported by the National Science Foundation Graduate Research Fellowship under Grant Number NSF DGE 0937373 and National Science Foundation I.G.E.R.T. under Grant Number NSF DGE 0549115, and National Science Foundation Grant 1453276 awarded to Pernille Hemmer. 


\section{References}

Baddeley, A.D. \& Scott, D. (1971). Short-term forgetting in the absence of proactive interference Quarterly Journal of Experimental Psychology, 23, 275-283.

Barrouillet, P., Bernardin, S., \& Camos, V. (2004). Time constraints and resource sharing in adults' working memory spans. Journal of Experimental Psychology: General, 133, 83-100.

Bae, G.Y., Olkonnen, M., Allred, S., \& Flombaum, J. (2015). Why some colors appear more memorable than others: A model combining categories and particulars in color working memory. Journal of Experimental Psychology: General, 144, 744-763.

Bays, P., Catalao, R.F., \& Husain, M. (2009). The precision of visual working memory is set by allocation of a shared resource. Journal of Vision, 9, 7-11.

Bays, P.M., Wu, E.Y., \& Husain, M. (2011). Storage and binding of object features in visual working memory. Neuropsychologia, 49, 1622-1631.

Berlin, B. \& P. Kay (1969). Basic Color Terms: Their Universality and Evolution. Berkeley: University of California Press.

Brady, T. F., Konkle, T., Gill, J., Oliva, A., \& Alvarez, G. A. (2013). Visual long-term memory has the same limit on fidelity as visual working memory. Psychological Science, 24, 981990.

Devkar, D. \& Wright, A. (2016). "Event-based proactive interference in rhesus monkeys". Psychonomic Bulletin \& Review, 1-9.

Donkin, C., Nosofksy, R., Gold, J., \& Shiffrin, R. (2014). Verbal labeling, gradual decay, and sudden death in visual short-term memory. Psychological Bulletin and Review, 21, 2-11. 
Fawcett, J. \& Lawrence, M., \& Taylor, T. (2016). The representational consequences of intentional forgetting: Impairments to both the probability and fidelity of long-term memory. Journal of Experimental Psychology: General, 145, 56-81.

Fischer, J. \& Whitney, D. (2014). “Serial dependence in visual perception”. Nature Neuroscience, $17,738-743$.

Fraley, C. \& Raftery, A.E. (2006). “MCLUST version 3 for R: normal mixture modeling and model-based clustering". (Technical report no. 504, Department of Statistics, University of Washington).

Gelman, A., Carlin, J. B., Stern, H. S., \& Rubin, D. B. (2003). Bayesian data analysis. Boca Raton, FL: Chapman \& Hall.

Griffiths, T.L. \& Tenenbaum, J.B. (2006). Optimal predictions in everyday cognition. Psychological Science, 17, 767-773.

Hemmer, P. \& Persaud, K. (2014). Interactions between categorical knowledge and episodic memory across domains. Frontiers in Psychology, 5, 1-18.

Hemmer, P. \& Steyvers, M. (2009a). Integrating Episodic Memories and Prior Knowledge at Multiple Levels of Abstraction. Psychonomic Bulletin \& Review, 16, 80-87.

Hemmer, P. \& Steyvers, M. (2009b). A Bayesian Account of Reconstructive Memory. Topics in Cognitive Science, 1, 189-202.

Hemmer, P., Tauber, S., \& Steyvers, M. (2015). Moving beyond qualitative evaluations of Bayesian models of cognition. Psychonomic Bulletin \& Review, 22, 614-628.

Huttenlocher, J., Hedges, L. V., \& Duncan, S. (1991). Categories and particulars: Prototype effects in establishing spatial location. Psychological Review, 98, 352-376. 
Huttenlocher, J., Hedges, L.V., \& Vevea, J.L. (2000). Why Do Categories Affect Stimulus Judgment? Journal of Experimental Psychology, 129, 220-241.

Lew, T.F., Pashler, H.E., \& Vul, E. (2015). Fragile associations coexist with robust memories for precise details in long-term memory. Journal of Experiment Psychology: Learning, Memory, and Cognition. Advance Online Publication.

Lewandowsky, S., Oberauer, K., \& Brown, G. (2009). No temporal decay in visual short-term memory. Trends in Cognitive Sciences, 13, 120-126.

Neath, I. \& Brown, G. (2012). Arguments against memory trace decay: a SIMPLE account of Baddeley and Scott. Frontiers in Psychology, 3, 1-3.

Persaud, K. \& Hemmer, P. (2014). The influence of knowledge and expectations for color on episodic memory. In P. Bello, M. Guarini, M. McShane, \& B. Scassellati (Eds.), Proceedings of the 36th Annual Conference of the Cognitive Science Society (pp. 11621167). Quebec City, CA: Cognitive Science Society.

Hemmer, P., Persaud, K., Kidd, C., \& Piantidosi, S. (2015). Shifting priors: Evaluating the crosscultural influence of color expectations on episodic memory. In Noelle, D. C., Dale, R., Warlaumont, A. S., Yoshimi, J., Matlock, T., Jennings, C. D., \& Maglio, P. P. (Eds.), Proceedings of the 37th Annual Meeting of the Cognitive Science Society. Austin, TX: Cognitive Science Society.Portrat, S., Barrouillet, P., \& Camos, V. (2008). Time-related decay or interference - based forgetting in working memory? Journal of Experimental Psychology: Learning, Memory, and Cognition, 34, 1561-1564.

Shiffrin, R.M., \& Steyvers, M. (1997). A model for recognition memory: REM: Retrieving Effectively from Memory. Psychonomic Bulletin \& Review, 4, 145-166. 
Spiegelhalter, D. J., Best, N. G., Carlin, B. R., \& van der Linde, A. (2002). Bayesian measures of model complexity and fit. Journal of the Royal Statistical Society Series B-Statistical Methodology, 64, 583-616.

Steyvers, M., \& Griffiths, T. L. (2008). Rational Analysis as a Link between Human Memory and Information Retrieval. In N. Chater \& M. Oaksford (Eds.), The Probabilistic Mind: Prospects from Rational Models of Cognition (pp. 327-347). Oxford: Oxford University Press.

Steyvers, M., Griffiths, T.L., \& Dennis, S. (2006). Probabilistic inference in human semantic memory. Trends in Cognitive Sciences, 10, 327-334.

Suchow, J. W., Brady, T.F., Fougnie, D., \& Alvarez, G. A. (2013). Modeling visual working memory with the MemToolbox. Journal of Vision, 13, 1-8.

van den Berg, R., Shin, H., Chou, W., George, R., \& Ma, W.J. (2012). Variability in encoding precision accounts for visual short-term memory limitations. Proceedings of the National Academies of Science, 109, $8780-8795$.

van der Linde, A. (2005). DIC in variable selection. Statistica Neerlandica, 1, 45-56.

Zhang, W., \& Luck, S.J. (2008). Discrete fixed-resolution representations in visual working memory. Nature, 453, 233-235. 


\section{Appendix}

\section{A.1 Label vs No-Label Parameters}

Table A1 gives the parameter values for the three experimental conditions: Label First (participants recall a color label prior to generating the color), Label Last (participants generated a color before providing a color label), and No Label (participants never provided a color label). For some lag groups, the model had a difficult time converging given the sparsity of the data. Also, there was no lag of 1 in the No label condition.

Table A1. Parameter estimates and (confidence intervals) for label vs. no-label conditions

\begin{tabular}{|c|c|c|c|c|c|c|}
\hline & \multicolumn{2}{|c|}{ Label First } & \multicolumn{2}{|c|}{ Label Last } & \multicolumn{2}{|c|}{ No Label } \\
\hline & Fidelity $\sigma\left(^{\circ}\right)$ & Guess $g$ & Fidelity $\sigma\left(^{\circ}\right)$ & Guess $g$ & Fidelity $\sigma\left(^{\circ}\right)$ & Guess $g$ \\
\hline Lag 1 & $12.19(11.09-13.97)$ & $\mathbf{0 . 0 4}(0.01-0.08)$ & $9.7(7.93-14.36)$ & $0.08(0.02-0.20)$ & - & - \\
\hline Lag 2-3 & $13.17(10.99-17.89)$ & $\mathbf{0 . 3 3}(0.23-0.42)$ & $8.9(6.8-18.32)$ & $0.4(0.21-0.55)$ & $17.46(15.23-20.49)$ & $\mathbf{0 . 4 5}(0.39-0.51)$ \\
\hline Lag 4-9 & $\mathbf{1 5 . 2 4}(12.56-19.49)$ & $\mathbf{0 . 4 6}(0.38-0.53)$ & $10.9(8.88-14.17)$ & $\mathbf{0 . 4}(0.31-0.54)$ & $19.96(18.10-22.79)$ & $\mathbf{0 . 4 5}(0.452-0.533$ \\
\hline Lag 10+ & $15.33(12.56-19.49)$ & $\mathbf{0 . 4 6}(0.38-0.53)$ & $29.84(14.69-93.48)$ & $0.61(0.02-0.83)$ & - & - \\
\hline All & $\mathbf{1 3 . 6 5}(12.40-15.36)$ & $\mathbf{0 . 4 0}(0.36-0.44)$ & $\mathbf{1 0 . 4 1}(8.64-12.97)$ & $\mathbf{0 . 4 3}(0.35-0.50)$ & $18.61(16.94-20.79)$ & $\mathbf{0 . 4 8}(0.447-0.515$ \\
\hline
\end{tabular}

\section{A.2 Parameters at All Lags}

Table A2. Parameters for each lag

\begin{tabular}{lcc}
\hline & Guessing rate & Fidelity memory \\
& $\boldsymbol{g}$ & $\boldsymbol{\sigma}\left(^{\circ}\right)$ \\
\hline Lag 1 & .06 & 11.85 \\
\hline Lag 2 & .37 & 18.13 \\
\hline Lag 3 & .47 & 14.07 \\
\hline Lag 4 & 0.44 & 18.13 \\
\hline Lag 5 & 0.47 & 18.10 \\
\hline Lag 6 & 0.52 & 16.09 \\
\hline Lag 7 & 0.56 & 17.14 \\
\hline Lag 8 & 0.49 & 18.04 \\
\hline Lag 9 & 0.54 & 19.38 \\
\hline Lag 10 & 0.63 & 14.78 \\
\hline Lag 11 & 0.48 & 10.37 \\
\hline All & 0.46 & 15.84 \\
\hline
\end{tabular}


To develop reasonable lag groups we first infer parameter values for all lags using the RG model. Table A2 provides RG model parameter values for each individual lag.

\section{A.3 RG model 0-360}

For comparison to the Bayesian Model on the full range of hue values, we implemented the

Table A3. RG Model Parameter Values (0-360)

\begin{tabular}{lcc}
\hline & Remember-Guess Model (RG) \\
\hline & $\begin{array}{c}\text { Fidelity (Conf. Int.) } \\
\boldsymbol{\sigma}\left({ }^{\circ}\right)\end{array}$ & $\begin{array}{c}\text { Guess Rate(Conf. Int. }) \\
\boldsymbol{g}\end{array}$ \\
\hline Lag 1 & $\mathbf{1 1 . 8 1}(10.59-13.26)$ & $\mathbf{0 . 0 6}(0.03-.010)$ \\
Lag 2-3 & $\mathbf{1 6 . 0 8}(14.40-18.15)$ & $\mathbf{0 . 4 2}(0.37-0.46)$ \\
Lag 4-9 & $\mathbf{1 7 . 6 8}(16.05-19.40)$ & $\mathbf{0 . 4 9}(0.46-0.53)$ \\
Lag 10+ & $\mathbf{1 5 . 1 3}(12.03-20.65)$ & $\mathbf{0 . 6 1}(0.53-0.69)$ \\
All & $\mathbf{1 5 . 8 3}(14.82-16.95)$ & $\mathbf{0 . 4 6}(0.44-0.48)$ \\
\hline
\end{tabular}

RG model on the same response data space (0-360 degree). There is no difference in the parameters between the two fittings of the RG model. See Table A3 for the inferred parameters.

\section{A.4 3CRG model without independent noise parameters}

An alternate version of the 3CRG model where the weighting $w=\psi^{2} /\left[\tau^{2}+\psi^{2}\right]$, but $\sigma_{\text {mem }}$ as the noise on one von Mises is replaced with $\psi$, was implemented. Instead of the noise component in one von Mises distribution being dependent on the other, here the noise parameters were treated as independent.

The probability density function of the 3CRG model with all inferred parameters is given by,

$$
\begin{gathered}
(1-g) *((1-w) * \operatorname{von} \operatorname{Mises}(0, \tau)+w * \operatorname{von} \operatorname{Mises}(0, \psi))+ \\
g * \operatorname{Unif}(0,360)
\end{gathered}
$$


Table A4. Hierarchical 3CRG Model with independent noise parameters

\begin{tabular}{|c|c|c|c|c|c|c|}
\hline \multicolumn{7}{|c|}{3 Component Remember Guess with independent noise parameters } \\
\hline & $\begin{array}{c}\text { Fidelity } \\
\sigma\left({ }^{\circ}\right)\end{array}$ & $\begin{array}{c}\text { Fidelity } \\
t\left({ }^{\circ}\right)\end{array}$ & $\begin{array}{c}\text { Guess Rate } \\
\quad g\end{array}$ & $\begin{array}{l}\text { Mixing } \\
\quad w\end{array}$ & AIC & BIC \\
\hline Lag 1 & $\mathbf{1 0 . 6 2 4}(9.41-12.74)$ & $28.55(17.05-80.52)$ & $\mathbf{0 . 0 3}(0.007-0.082)$ & $\mathbf{0 . 5 6}(0.26-0.93)$ & 1657.81 & 1673.20 \\
\hline Lag 2-3 & $13.77(12.30-16.15)$ & $29.46(23.06-44.79)$ & $\mathbf{0 . 4 1}(0.35-0.45)$ & $\mathbf{0 . 4 5}(0.33-0.62)$ & 9252.46 & 9272.29 \\
\hline Lag 4-9 & $15.30(13.65-16.83)$ & $31.26(23.61-35.52)$ & $\mathbf{0 . 4 8}(0.45-0.52)$ & $\mathbf{0 . 4 4}(0.31-0.60)$ & 17077.14 & 17098.72 \\
\hline Lag 10+ & $14.02(12.28-581.89)$ & $31.06(11.93-989.33)$ & $\mathbf{0 . 5 8}(0.53-0.69)$ & $\mathbf{0 . 4 5}(0.36-0.97)$ & 3183.15 & 3199.61 \\
\hline All & $\mathbf{1 3 . 8 6}(13.01-15.07)$ & $\mathbf{2 9 . 6 0}(25.04-39.40)$ & $\mathbf{0 . 4 4}(0.41-0.47)$ & $\mathbf{0 . 4 8}(0.38-0.58)$ & 31339.02 & 31362.48 \\
\hline
\end{tabular}

where $g, \psi$ and $\tau$ are all inferred values from the data. Table A4 shows the parameter values for each lag group under this model and reports the AIC and BIC scores relative to the RG model. The parameter values for this model in the hierarchical fitting detailed in the modeling section (see section 4.2) were identical to the individual lag fitting and are not reported. Note that the parameter values at lag 10+ had a tendency to reverse in different runs of the hierarchical model, such that sometimes $\psi \approx 14$ and $\tau \approx 30$, but at other times $\psi \approx 30$ and $\tau \approx 14$. Irrespective of the order of the parameter values this version of the 3CRG model consistently had the lowest $\mathrm{DIC}=31177.53$ in the hierarchical implementation of this model, which favors this model relative to both the RG and BM models.

\section{A.5 $3 C R G$ model with all parameters inferred}

The 3CRG model with all the model parameters inferred was implemented. Instead of the noise component in one von Mises distribution being dependent on the other, here the noise parameters were treated as independent and $w$ is an additional free parameter.

The probability density function of the $3 \mathrm{CRG}$ model with all inferred parameters is given by,

$$
\begin{gathered}
(1-g) *((1-w) * \operatorname{von} \operatorname{Mises}(0, \tau)+w * \operatorname{von} \operatorname{Mises}(0, \psi))+\operatorname{Eq}(\mathrm{A} 2) \\
g * \operatorname{Unif}(0,360)
\end{gathered}
$$


Table A5. 3CRG Model with all parameters inferred, and with AIC and BIC Scores

\begin{tabular}{|c|c|c|c|c|c|c|}
\hline \multicolumn{7}{|c|}{3 Component Remember Guess $_{\text {all }}$ (3CRG) } \\
\hline & $\begin{array}{c}\text { Fidelity } \\
\sigma\left({ }^{\circ}\right)\end{array}$ & $\begin{array}{c}\text { Fidelity } \\
t\left({ }^{\circ}\right)\end{array}$ & $\begin{array}{c}\text { Guess Rate } \\
g\end{array}$ & $\begin{array}{c}\text { Mixing } \\
\quad w\end{array}$ & AIC & BIC \\
\hline Lag 1 & $6.97(5.02-11.84)$ & $19.95(13.43-47.71)$ & $\mathbf{0 . 0 3}(.008-.082)$ & $0.56(0.26-0.93)$ & 1657.87 & 1678.39 \\
\hline Lag 2-3 & 8.45 (6.66-11.27) & $28.40(23.23-46.63)$ & $\mathbf{0 . 3 6}(0.30-0.41)$ & $\mathbf{0 . 4 5}(0.33-0.62)$ & 9229.60 & 9256.03 \\
\hline Lag 4-9 & $9.57(7.66-12.45)$ & $31.05(24.76-44.41)$ & $\mathbf{0 . 4 3}(0.38-0.48)$ & $\mathbf{0 . 4 4}(0.31-0.60)$ & 17051.95 & 17080.72 \\
\hline Lag 10+ & $13.07(10.37-19.02)$ & $94.68(25.88-439.98)$ & $0.28(0.03-0.65)$ & $\mathbf{0 . 4 5}(0.36-0.97)$ & 3312.54 & 3334.49 \\
\hline All & 9.04 (7.71-10.63) & $29.64(24.96-39.28)$ & $\mathbf{0 . 4 0}(0.36-0.43)$ & $\mathbf{0 . 4 8}(0.38-0.58)$ & 1657.87 & 31314.45 \\
\hline
\end{tabular}

Table A6. Hierarchical 3CRG Model with all parameters inferred

\begin{tabular}{ccccc}
\hline \multicolumn{5}{c}{ Hierarchical 3CRG all } \\
\hline & $\begin{array}{c}\text { Fidelity } \\
\boldsymbol{\sigma}\left({ }^{\circ}\right)\end{array}$ & $\begin{array}{c}\text { Prior } \\
\boldsymbol{\tau}\left({ }^{\circ}\right)\end{array}$ & $\begin{array}{c}\text { Guess Rate } \\
\boldsymbol{g}\end{array}$ & $\begin{array}{c}\text { Mixing } \\
\boldsymbol{w}\end{array}$ \\
\hline Lag1 & $\mathbf{7 . 0 6}(6.40-13.61)$ & $\mathbf{1 9 . 5 0}(6.86-140.95)$ & $\mathbf{0 . 0 4}(0.01-0.12)$ & $\mathbf{0 . 5 6}(0.38-0.97)$ \\
Lag 2-3 & $\mathbf{8 . 3 8}(8.38-14.26)$ & $\mathbf{2 8 . 6 5}(28.65-149.05)$ & $\mathbf{0 . 3 6}(0.01-0.38)$ & $\mathbf{0 . 4 5}(0.45-0.83)$ \\
Lag 4-9 & $\mathbf{9 . 6 8}(8.58-15.99)$ & $\mathbf{3 1 . 6 8}(20.68-131.25)$ & $\mathbf{0 . 4 4}(0.29-0.53)$ & $\mathbf{0 . 4 4}(0.28-0.86)$ \\
Lag 10+ & $\mathbf{1 3 . 1 9}(.25-15.45)$ & $\mathbf{1 1 4 . 6 8}(21.95-114.68)$ & $\mathbf{0 . 0 0}(0.00-0.63)$ & $\mathbf{0 . 3 3}(0.12-0.86)$ \\
\hline
\end{tabular}

where $g, w, \psi$ and $\tau$ are all inferred values from the data. Table A5 shows the parameter values for each lag group under this model and reports the AIC and BIC scores relative to the RG model. Table A6 shows the parameter values for this model in the hierarchical fitting detailed in the modeling section (see section 4.2). Note that the parameter values at lag $10+$ changes between the two implementations of this model. The DIC score of the hierarchical version of this model was 31194.54, which still favors this model relative to both the RG and BM models.

\section{A.6 Cluster analysis}




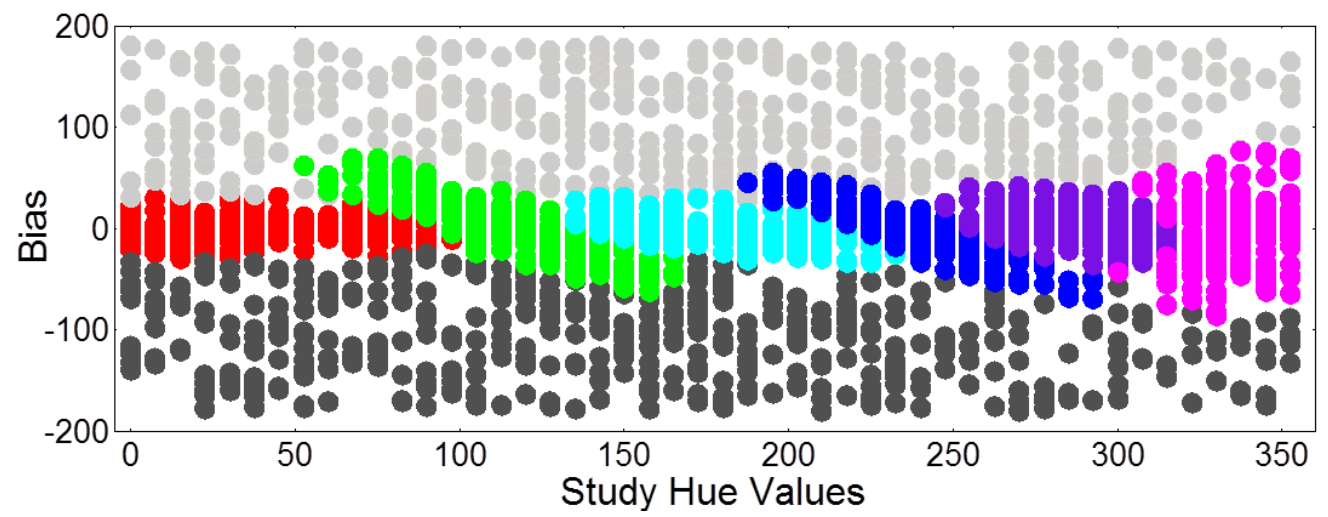

Figure A1. The 8-group unconstrained model-based classification of the data. 8 optimal clusters were learned from the Expectation-Maximization algorithm evaluated by BIC scores. Each of the 8 colors correspond to a different cluster that is color coded to reflect the color category to which most of the study values in the data belong.

The cluster analysis was implemented to infer the categories participants regressed to in the experiment. Briefly, the clustering algorithm (Fraley \& Raftery, 2006) performs a hierarchical agglomeration to maximize the classification likelihood for up to 9 groups in each model. Next, the Expectation-Maximization (EM) algorithm calculates the maximum likelihood estimation for all models and number of cluster combinations. Lastly, the algorithm computes the BIC scores for each cluster mixture model with optimal parameter values and returns the best fitting cluster size model. The best BIC score $(\mathrm{BIC}=-66210.13)$ revealed that 8 clusters produced the most optimal partitioning of the data. Figure A1, shows the output from the learned clusters. The mean of the 8 inferred clusters (in degrees) were: $39.59,151.76,171.21,113.88,182.15,238.42$, 288.69, and 335.57). 